\title{
Left atrial strain measured by 4D Auto LAQ echocardiography is significantly correlated with high risk of thromboembolism in patients with non-valvular atrial fibrillation
}

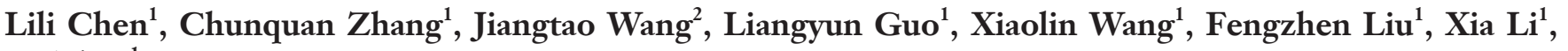 \\ Yu Zhao ${ }^{1}$ \\ ${ }^{1}$ Department of Ultrasound, Second Affiliated Hospital of Nanchang University, Nanhang, China; ${ }^{2}$ GE Clinical Education Team, Beijing, China \\ Correspondence to: Chunquan Zhang, Department of Ultrasound, the Second Affiliated Hospital of Nanchang University, 1 Minde Road, Donghu \\ District, Nanchang 330006, China. Email: jxzcq@163.com.
}

Background: The 4-dimensional automated left atrial quantification (4D Auto LAQ) tool is a new software for analysis of the structure and function of the left atrium (LA). This study aimed to evaluate the relationship between LA strain (LAS) as measured by 4D Auto LAQ echocardiography and thromboembolism risk in patients with non-valvular atrial fibrillation (NVAF).

Methods: Eight-five patients with NVAF were recruited from the cardiovascular center of our hospital, including 39 patients at high risk and 46 patients at low risk of thromboembolism. The study participants were assessed by routine echocardiography; 4D images were obtained, after which 4D Auto LAQ assessment was performed.

Results: In the thromboembolism high-risk group, the rates of impaired LA reservoir strain, LA contraction strain, LA reservoir circumferential strain, LA conduit circumferential strain, and LA contraction circumferential strain were found to be significantly higher than in the low-risk group. However, there was no significant difference in volume at onset of LA contraction or LA ejection fraction (LAEF) between the 2 groups. LA contraction circumferential strain was found to be an independent high risk factor for thromboembolism [odds ratio (OR): 2.52; $\mathrm{P}=0.008$ ]. LA contraction circumferential strain $>-4.5 \%$ was the cut-off for differentiating between participants with high and low risk of thromboembolism, with an area under the curve (AUC) of $0.95(\mathrm{P}<0.0001)$, a sensitivity of 0.872 , and a specificity of 0.978 . Sequential analysis revealed that LA contraction circumferential strain had a high diagnostic efficacy for stroke, as well as a specified accuracy in the diagnosis of hypertension and diabetes in patients aged $\geq 65$ years old. However, it was not found to be effective in the diagnosis of heart failure and vascular diseases.

Conclusions: LAS is a useful index for the dynamic evaluation of LA function in patients with nonvalvular AF, with higher sensitivity and accuracy than LA volume. LA contraction circumferential strain is an independent high risk factor for thromboembolism, and LA contraction circumferential strain $>-4.5 \%$ is a valuable cut-off to guide the use of anticoagulant therapy in patients with non-valvular AF.

Keywords: Non-valvular atrial fibrillation (NVAF); 4-Dimensional Automated Left Atrial Quantification (4D Auto LAQ); left atrial strain (LAS); $\mathrm{CHA}_{2} \mathrm{DS}_{2}-\mathrm{VAS}_{\mathrm{C}}$ score

Submitted Dec 21, 2020. Accepted for publication Apr 08, 2021.

doi: 10.21037 /qims-20-1381

View this article at: http://dx.doi.org/10.21037/qims-20-1381 


\section{Introduction}

Atrial fibrillation (AF), the most common form of persistent arrhythmia, has been found to increase the risk of ischemic stroke by a factor of 4 to 5 on average while increasing cardiac mortality by a factor of 2 (1). This condition endangers patients' lives and greatly affects their life quality (2). The prevention of complications related to AF, such as stroke, is an important component in its comprehensive management. The $\mathrm{CHA}_{2} \mathrm{DS}_{2}$-VASc [congestive heart failure, hypertension, age $\geq 75$ years, diabetes mellitus, stroke or transient ischemic attack (TIA), vascular disease, age 65 to 74 years, sex category] score is the most common method for stratifying thromboembolism risk in patients with $\mathrm{AF}$, and it is also used to inform decisions on anticoagulation therapy. As men with $\mathrm{CHA}_{2} \mathrm{DS}_{2}$-VASc scores $\geq 2$ and women with $\mathrm{CHA}_{2} \mathrm{DS}_{2}$-VASc scores $\geq 3$ are high-risk groups for thromboembolism, anticoagulant therapy should be considered for these patients $(3,4)$. However, this score is only based on clinical risks and does not take into account the structure or function of the left atrium (LA). Studies have reported that AF can aggravate LA remodeling, including LA structure, function, as well as electrophysiological remodeling $(5,6)$. Moreover, LA remodeling is closely correlated with ischemic stroke $(7,8)$.

LA strain (LAS) measured by speckle tracking echocardiography (STE) has been used by many scholars to study LA function $(9,10)$. However, despite the value of STE $(11,12)$, its clinical applications are limited by the thinwalled structure of the LA, irregular arrangement of the LA myocytes (13), and intervendor variability (14).

The 4-Dimensional Automated Left Atrial Quantification (4D Auto LAQ) tool is an LA analysis technique that uses $3 \mathrm{D}$ volume data to determine the volume and LA ejection fraction (LAEF) as well as LA longitudinal and circumferential strains. The present study aimed to evaluate the correlation between LAS, as measured by 4D Auto LAQ, and thromboembolism risk, as defined by $\mathrm{CHA}_{2} \mathrm{DS}_{2}$-VASc scores in patients with non-valvular atrial fibrillation (NVAF). Moreover, we quantitatively defined the LAS parameters that are independent high risk factors for thromboembolism, and established their cut-off points so as to inform decisions on clinical anticoagulation therapy in patients with NVAF.

\section{Methods}

\section{Study population}

This single-center retrospective study obtained ethical approval from the Second Affiliated Hospital of Nanchang University. The procedures used conformed to the standards of the World Medical Association Declaration of Helsinki (as revised in 2013). Between August 2019 and June 2020, 136 patients with AF were admitted to the Department of Cardiology of the Second Affiliated Hospital of Nanchang University. Patients with NVAF who did not have moderate-to-severe mitral stenosis or the presence of mechanical prosthetic heart valves were enrolled in the study. After relevant examinations, $\mathrm{CHA}_{2} \mathrm{DS}_{2}-\mathrm{VASc}$ assessment was performed by experienced cardiologists. Among the participants recruited, 51 patients were excluded due to having atrial septal defect $(\mathrm{n}=5)$, valvular disease $(n=7)$, or poor-quality echocardiographic images $(n=16)$. Patients with NVAF who were not in sinus rhythm during imaging were also excluded ( $\mathrm{n}=23)$. Eventually, 85 patients with NVAF were included in the study.

\section{Embolism risk score}

Comorbid conditions such as heart failure, stroke, diabetes, age, female sex, hypertension, and vascular disease were considered when risk-stratifying the participants for embolism according to the $\mathrm{CHA}_{2} \mathrm{DS}_{2}$-VASc criteria. One point each was assigned for hypertension, congestive heart failure, diabetes, vascular disease (peripheral arterial disease and prior myocardial infarction), age (65-74 years), and female sex. Two points each were assigned for age $>75$ years, systemic embolism, stroke, and TIA. Based on the score, participants were allocated into either the thromboembolism high-risk group ( $\mathrm{n}=39$; males with $\mathrm{CHA}_{2} \mathrm{DS}_{2}-\mathrm{VASC} \geq 2$ and females with $\mathrm{CHA}_{2} \mathrm{DS}_{2}$-VASC $\geq 3$ ) or the low-risk group $\left(\mathrm{n}=46\right.$; males with $\mathrm{CHA}_{2} \mathrm{DS}_{2}$-VASC $<2$ and females with $\mathrm{CHA}_{2} \mathrm{DS}_{2}$-VASC $<3$ ). Participants were examined by routine echocardiography and 4D Auto LAQ analysis.

\section{Image acquisition}

The GEvivid E95 ultrasonic diagnostic instrument (GE Healthcare; Vingmed Ultrasound, Horten, Norway) with an M5S probe (frequency: 1.5-4.6 MHz) and $4 \mathrm{~V}$ probe (frequency: $1.5-4.0 \mathrm{MHz}$ ) was used for imaging. All participants underwent a complete transthoracic echocardiogram examination with the M5S transducer to determine cardiac structure, chamber size, mitral regurgitation, and cardiac function according to the current guidelines of the American Society of Echocardiography (15). 

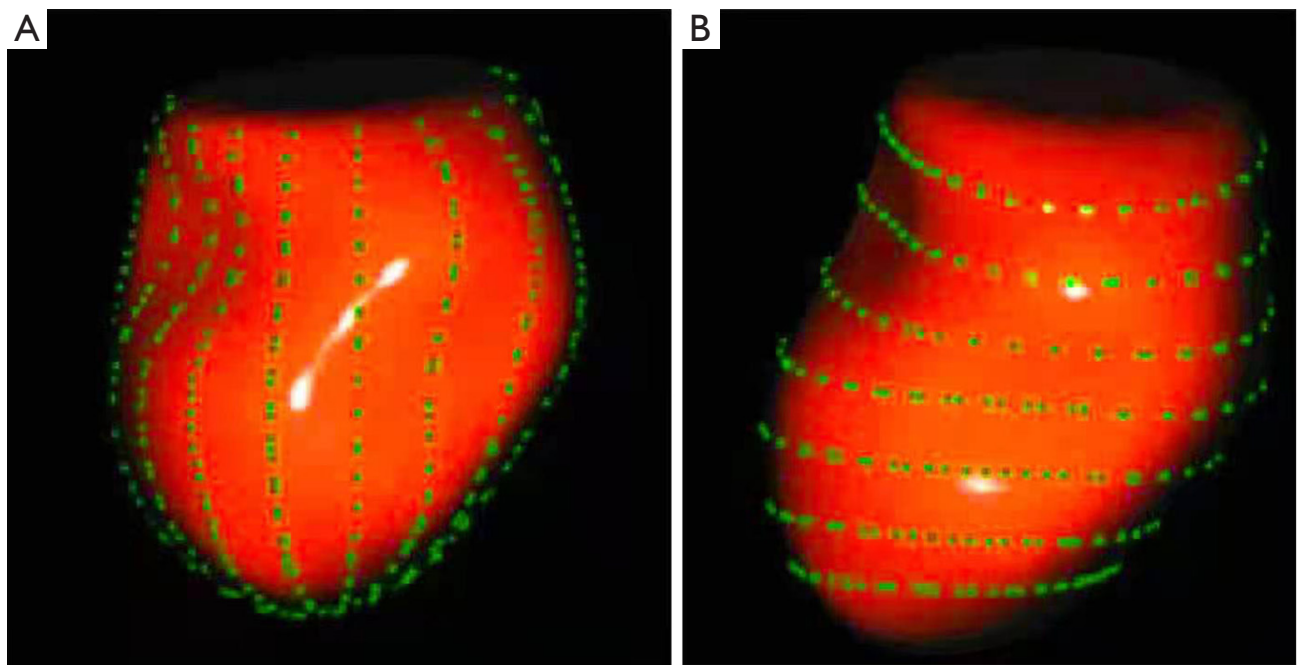

Figure 1 Schematic diagram of measuring left atrial strain by the 4-Dimensional Automated Left Atrial Quantification (4D Auto LAQ) tool. (A) The longitudinal line points for longitudinal strain calculation; (B) the circumferential line points for circumferential strain calculation.

The left ventricular ejection fraction (LVEF) was measured by the improved biplane Simpson method. All participants then underwent $3 \mathrm{D}$ echocardiographic examination using a $4 \mathrm{~V}$ transducer. The image was optimized to obtain the full volume data of the whole LA under the apical 4-chamber view. Five heartbeat acquisition settings were used to obtain the dataset, and the frame rate was adjusted to $40 \%$ of the heart rate. Participants were encouraged to hold their breath at the end of breath (either 'inhalation' or 'exhalation') when feasible. At least 4 datasets were obtained for each participant, with the 3 best-quality data sets being selected for offline analysis. Datasets with part of the LA missing, blurred endocardial boundaries, or obvious suture artifacts were excluded.

During echocardiographic acquisition, the patients were in sinus rhythm. High-quality images were collected, so as to enhance the accuracy and repeatability of the measurements.

\section{Principles of 4D Auto LAQ}

A semi-automated segmentation algorithm was used for volume calculation. The algorithm was initialized with 1 landmark located in the center of the mitral valve (MV) at the annulus level. The segmentation algorithm computes the deformation of the 3D model by solving a state estimation problem using an extended Kalman filter that combines the LA motion model, geometry, and edge detection algorithms. Strain was calculated based on changes in the lengths of different lines along each anatomical direction. To calculate longitudinal strain, 8 longitudinal lines (Figure $1 A$ ), each connecting 2 opposite LA basal points, were sampled from an automatically constructed triangular mesh. To calculate circumferential strain, 7 circumferential lines (Figure $1 B$ ) that were equidistantly distributed between the LA base and the LA apex were used. The strain time was then calculated for each frame $s(t)=(L(t)-L(t r)) / L(t r) x$ $100 \%$, in which $\mathrm{L}(\mathrm{t})$ is the line length at time $\mathrm{t}$ and $\operatorname{tr}$ is the time of the left ventricular end diastole (ED). Global strain was calculated as the average strain of the respective directional lines of each direction.

\section{D Auto LAQ analysis}

Images were imported into the EchoPAC203 software (GE Healthcare) and selected for analysis. The analysis mode was entered, and the volume and 4D Auto LAQ submode were sequentially selected. After that, the sampling point was placed in the middle of the mitral orifice in the 3 planes. The 'review' function was selected to obtain the LA parameters, including volume and strain parameters, measured by 4D Auto LAQ, (Figure 2 and Video 1).

Volume parameters were volume at onset of LA contraction (LA VpreA) and LAEF, while LAS parameters were longitudinal and circumferential strains. The reference point was set at the left ventricle ED. During the reservoir phase, the LA wall lengthened, so the strain in this phase had a positive value. In the other 2 phases, the LA wall 

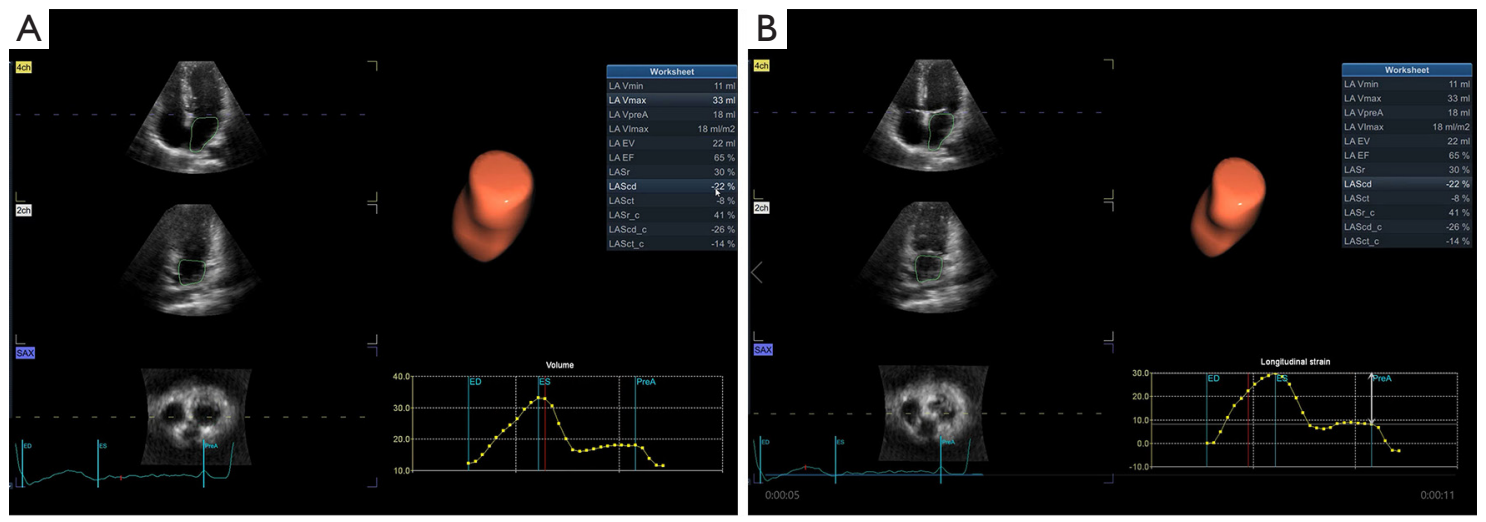

Figure 2 Left atrial volume curve and strain curve measured by the 4-Dimensional Automated Left Atrial Quantification (4D Auto LAQ) tool. (A) The volume curve; (B) The strain curves.

shortened, so the strains in these phases had negative values. LA longitudinal strain parameters obtained using this technique included LA reservoir strain (LASr), LA conduit strain (LAScd), and LA contraction strain (LASct), and LA circumferential strain parameters obtained included LA reservoir circumferential strain (LASr-c), LA conduit circumferential strain (LAScd-c), and LA contraction circumferential strain (LASct-c).

The longitudinal strain during the reservoir phase (LASr) is the difference of the strain value at $\mathrm{MV}$ opening minus ventricular $\mathrm{ED}$; the longitudinal strain during the conduit phase (LAScd) is the difference of the strain value at the onset of atrial contraction (PreA) minus MV opening; the longitudinal strain during the contraction phase (LASct) is the difference of the strain value at ventricular ED minus onset of atrial contraction; LASr-c is the difference of the strain value at MV opening minus ventricular ED; LAScd-c is the difference of the strain value at the onset of atrial contraction minus MV opening; LASct-c is the difference of the strain value at ventricular ED minus onset of atrial contraction; LA VpreA = Volume at onset of atrial contraction; LA EF = (Maximum atrial volume - Minimum atrial volume) / Maximum atrial volume.

\section{Statistical analysis}

Continuous variables were expressed as medians with $25-75 \%$ interquartile ranges (IQR) or means \pm standard deviations (SD) according to variable distribution. The Mann-Whitney $\mathrm{U}$ test or independent-samples $t$ test was used to compare medians or means between the 2 groups. The $\chi^{2}$ test was used for comparisons of the prevalence of comorbid conditions (diabetes, hypertension, degree of mitral regurgitation, and other medical illnesses) between the 2 groups. Logistic regression analysis was used to determine predictors of high risk of thromboembolism in patients with $\mathrm{AF}$. The receiver operating characteristic (ROC) curve was used to establish the critical value, which is the parameter value corresponding to the maximum Youden index value (Youden index $=$ sensitivity + specificity- 1 ). A $\mathrm{P}$ value $\leq 0.05$ was considered to be statistically significant. Statistical analyses were performed using SPSS version 23.0 (IBM Corp., Chicago, IL, USA).

\section{Results}

\section{Clinical characteristics}

Clinical characteristics of the thromboembolism highand low-risk groups are shown in Table 1. There were no significant differences in LVEF, body mass index (BMI), or the clinical course of NVAF between the 2 groups. The high-risk group had higher numbers of participants with hypertension, diabetes, stroke, and vascular disease than the low-risk group, but the differences in sex, heart failure, and mitral regurgitation between the 2 groups were not significant. Also, compared to the low-risk group, the highrisk group was more advanced in age.

\section{Univariate analysis of $4 D$ Auto $L A Q$}

Univariate analysis revealed that LASr $[6.00 \%(3.00-$ $12.00 \%)$ vs. $11.00 \%(7.75-18.00 \%) ; \mathrm{P}<0.001]$, LASr-c [5.00\% (2.00-9.00\%) vs. 11.00\% (6.75-21.25\%); $\mathrm{P}<0.001]$, and LAScd-c $[-7.00 \%(-12.00$ to $-2.00 \%)$ vs. $-1.50 \%$ 
Table 1 Baseline characteristics of study participants

\begin{tabular}{|c|c|c|}
\hline Demographic and clinical characteristics & \multicolumn{2}{|c|}{ Thromboembolism risk of NVAF patients } \\
\hline Age (years) (IQR) & 56.0 (50.8 to 62.5$)$ & $68.0(63.0 \text { to } 78.0)^{\star}$ \\
\hline LVEF (IQR) & 60.5 (55.75 to 67.00$)$ & 58.0 (52.0 to 64.0$)$ \\
\hline $\mathrm{BMI}($ mean $\pm \mathrm{SD})$ & $23.1 \pm 2.6$ & $22.8 \pm 3.0$ \\
\hline Male & 32 & 23 \\
\hline \multicolumn{3}{|l|}{ Comorbidities } \\
\hline Heart failure & 2 & 6 \\
\hline Hypertension & 6 & $29^{*}$ \\
\hline Vascular disease & 0 & $4^{*}$ \\
\hline \multicolumn{3}{|l|}{ Mitral regurgitation } \\
\hline Mild & 14 & 15 \\
\hline Moderate & 7 & 13 \\
\hline Severe & 0 & 0 \\
\hline None & 25 & 11 \\
\hline
\end{tabular}

*, $\mathrm{P}<0.05 . \mathrm{IQR}$, the values are median with $25-75 \%$ interquartile range; mean $\pm \mathrm{SD}$, the values are presented as mean \pm standard deviation; the rest of the values are represented as number of patients. NVAF, non-valvular atrial fibrillation; BMI, body mass index.

(-8.25-0.25\%); $\mathrm{P}=0.005]$ were lower in the high-risk group than in the low-risk group. However, LASct $[0.00 \%$ $(-2.00-3.00 \%)$ vs. $-7.00 \%$ ( -10.00 to $-4.75 \%) ; \mathrm{P}<0.001]$ and LASct-c $(1.59 \pm 5.80 \%$ vs. $-11.50 \pm 3.60 \% ; \mathrm{P}<0.001)$ were higher in the high-risk group than in the low-risk group $(\mathrm{P}<0.001)$. LA VpreA, LA EF, and LAScd did not differ significantly between the 2 groups (Table 2).

\section{Logistic regression analysis}

Logistic regression analysis of patients with $\mathrm{AF}$ revealed that LASct-c was independently associated with a high risk of thromboembolism [odds ratio (OR): 2.52, CI: 1.27 to 4.99, $\mathrm{P}=0.008$; Table 3 and Figure 3]. In patients with NVAF, LASct-c was found to be able to distinguish between high and low risk of thromboembolism, and the area under the curve (AUC) was $0.95(\mathrm{P}<0.001$; Figure 4$)$. The most approximate Youden index was 0.85 , while the corresponding value of LASct-c was $-4.5 \%$. At this point, sensitivity and specificity were 0.872 and 0.978 , respectively.

\section{Diagnostic efficacy of LASct-c for the factors in $\mathrm{CHA}_{2} \mathrm{DS}_{2^{-}}$ VASC}

To establish the predictive value of LASct-c for factors in $\mathrm{CHA}_{2} \mathrm{DS}_{2}$-VASC, LASct-c was used as the test variable, with or without congestive heart failure, hypertension, age ( $\geq 65$ years old), diabetes, stroke, and vascular disease as variables, and ROC curves were drawn. LASct-c was found to exhibit high diagnostic efficacy for stroke $(\mathrm{P}<0.001)$, with a sensitivity of 0.957 and a specificity of 0.823 . LASct-c exhibited a certain accuracy in the identification of hypertension, diabetes, and age $\geq 65$ years $(\mathrm{P}<0.001$, $0.036,<0.001$, respectively): the AUCs were 0.804, 0.726, and 0.765 , respectively; the sensitivity was $0.77,0.875$, and 0.676 , respectively; and the specificity was $0.84,0.64$, and 0.83 , respectively. LASct-c did not exhibit diagnostic efficacy for heart failure or vascular diseases ( $\mathrm{P}$ value: 0.102 and 0.378, respectively; AUC: 0.68 and 0.63 , respectively; Figure 5). As female sex is not considered to be a risk factor for thromboembolism in patients with $\mathrm{AF}(16)$, we did not 
Table 2 Univariate analysis 4D Auto LAQ-related parameters between the 2 groups

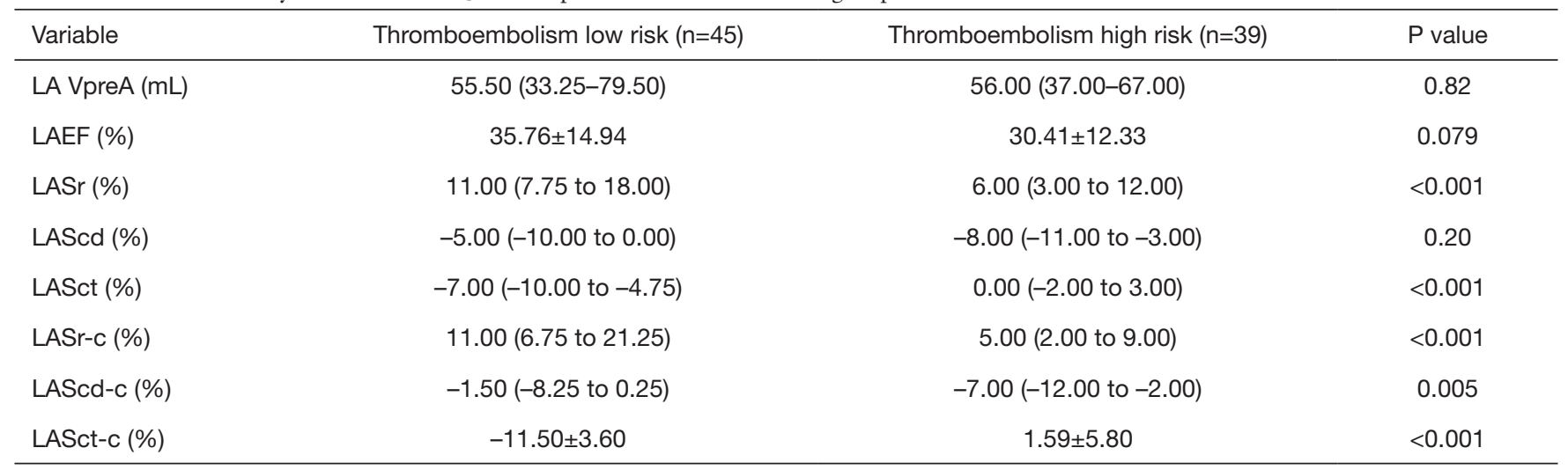

Data are given as median with $25-75 \%$ interquartile range or mean \pm standard deviation. LA VpreA, volume at onset of left atrial contraction; LA EF, left atrial ejection fraction; LASr, left atrial reservoir strain; LAScd, left atrial longitudinal strain during the conduit phase; LASct, left atrial contraction strain; LASr-c, left atrial reservoir circumferential strain; LAScd-c, left atrial conduit circumferential strain; LASct-c, left atrial contraction circumferential strain.

Table 3 Logistic regression analysis for the prediction of high risk of thromboembolism

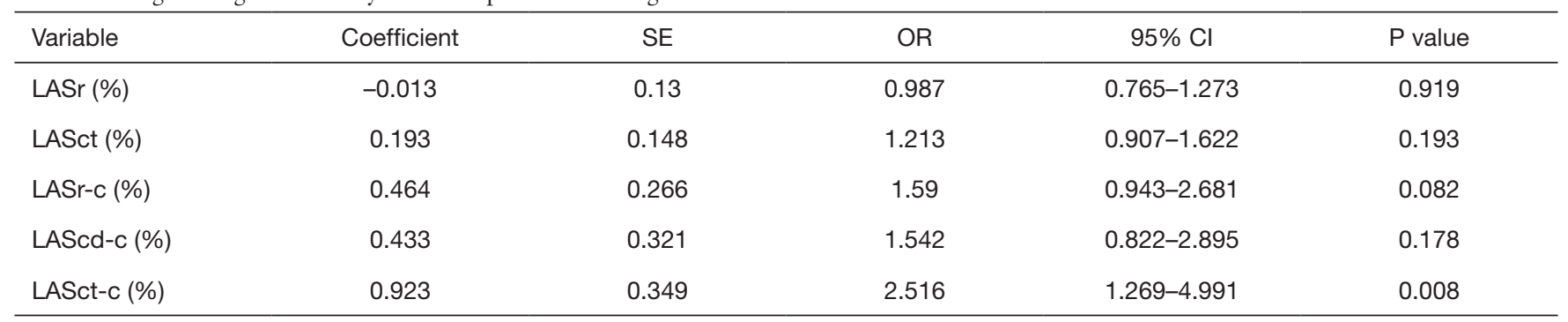

$\mathrm{Cl}$, confidence interval; OR, odds ratio; SE, standard error; LASr, left atrial reservoir strain; LASct, left atrial contraction strain; LASr-c, left atrial reservoir circumferential strain; LAScd-c, left atrial conduit circumferential strain; LASct-c, left atrial contraction circumferential strain.

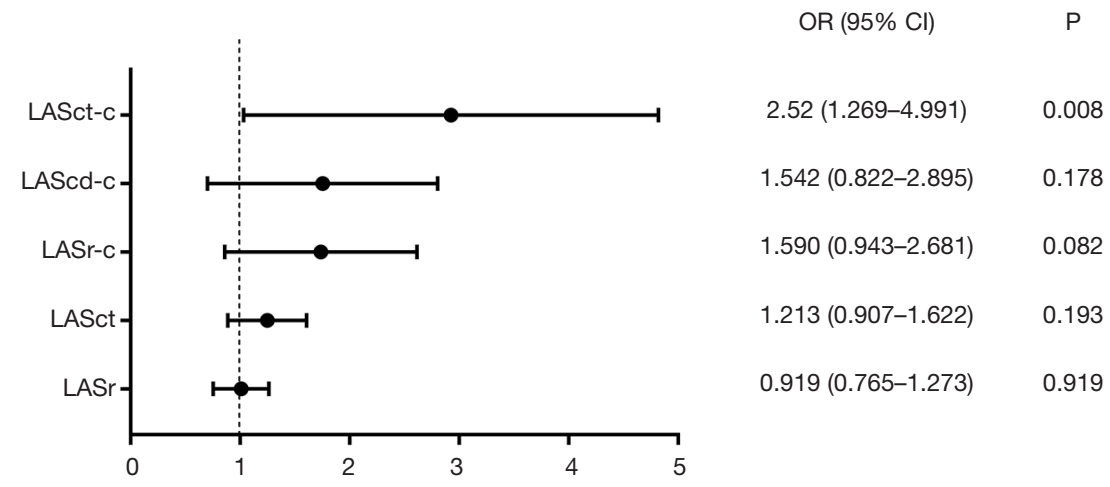

Figure 3 Logistic regression forest map of left atrial strain in non-valvular atrial fibrillation patients. OR, odds ratio; CI, confidence interval. 


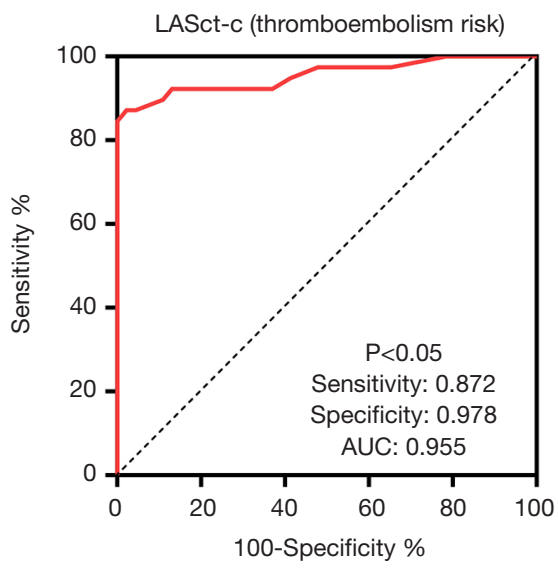

Figure 4 LASct-c ROC curve for predicting thromboembolism high risk in non-valvular atrial fibrillation patients. LASct-c, left atrial contraction circumferential strain; ROC, receiver operating characteristic; AUC, area under the curve. test the efficacy of LASCT-c in female participants.

\section{Reproducibility of $4 D$ Auto $L A Q$}

Repeat 4D Auto LAQ measurements of 15 randomly selected participants were taken by the same observers and by another experienced reader 2 weeks later, which allowed for the determination of intra-class correlation coefficients (ICC) as a measure of intra-observer and interobserver agreement. The ICC values ranged from 0.861 to 0.999 , (Table 4), indicating good interobserver and intraobserver repeatability.

\section{Discussion}

Compared to low-risk patients, patients at high risk of thromboembolism had significantly impaired LASr, LASct,
A

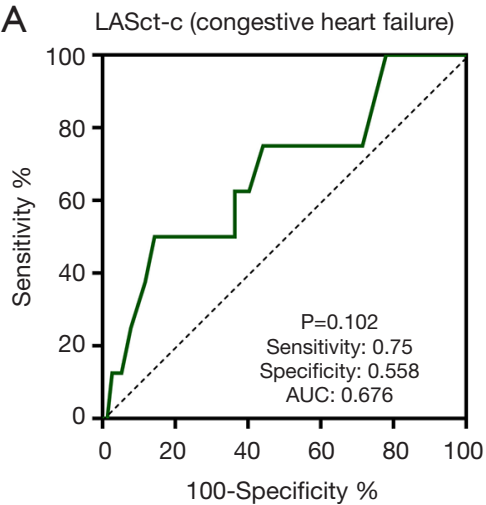

D

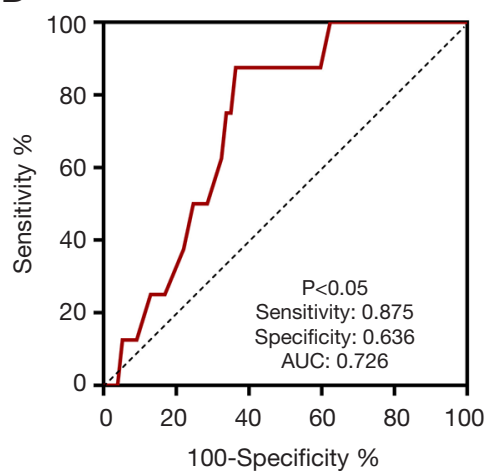

B

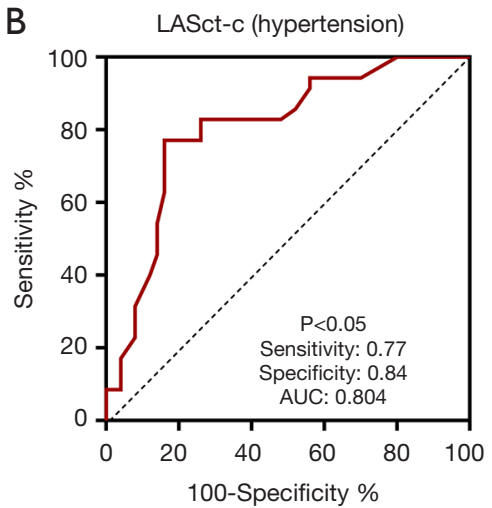

E

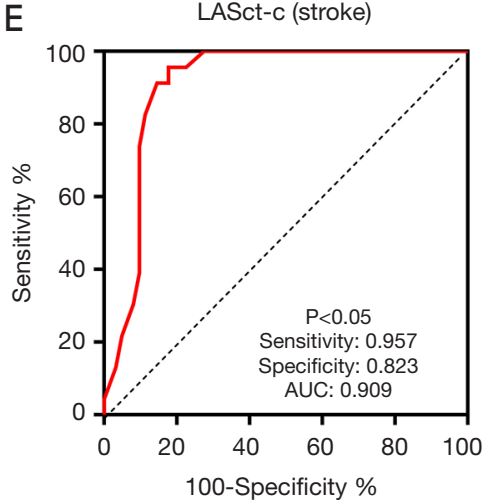

C

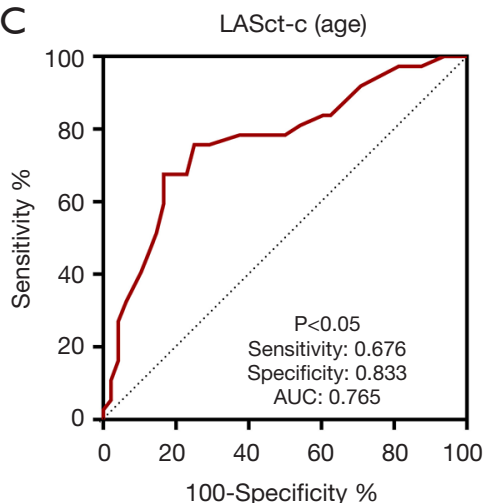

$\mathrm{F}$

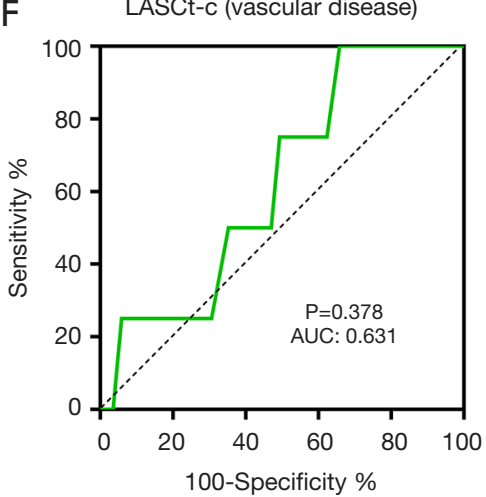

Figure 5 Diagnostic efficacy of LASct-c for the factors in $\mathrm{CHA}_{2} \mathrm{DS}_{2}-\mathrm{VAS}_{\mathrm{C}}$. (A) LASct-c ROC curve for predicting heart failure; (B) t LASct-c ROC curve for predicting hypertension; (C) LASct-c ROC curve for predicting age $\geq 65$ years old; (D) LASct-c ROC curve for predicting diabetes; (E) LASct-c ROC curve for predicting stroke and embolism; (F) LASct-c ROC curve for predicting vascular disease. LASct-c, left atrial contraction circumferential strain; ROC, receiver operating characteristic; AUC, area under the curve; $\mathrm{CHA}_{2} \mathrm{DS}_{2}-\mathrm{VAS}_{\mathrm{C}}$, congestive heart failure, hypertension, age $\geq 75$ years, diabetes mellitus, stroke or transient ischemic attack (TIA), vascular disease, age 65 to 74 years, sex category. 
Table 4 Interobserver and intraobserver variability

\begin{tabular}{|c|c|c|c|c|c|c|}
\hline Parameters & \multicolumn{3}{|c|}{ Interobserver variability } & \multicolumn{3}{|c|}{ Intraobserver variability } \\
\hline LAVpreA & 0.999 & $0.999-1.000$ & $<0.001$ & 0.998 & $0.993-0.995$ & $<0.001$ \\
\hline LAEF & 0.998 & $0.994-0.999$ & $<0.001$ & 0.997 & $0.990-0.999$ & $<0.001$ \\
\hline LASr & 0.996 & $0.989-0.999$ & $<0.001$ & 0.985 & $0.956-0.995$ & $<0.001$ \\
\hline LASct & 0.968 & $0.908-0.989$ & $<0.001$ & 0.951 & $0.861-0.983$ & $<0.001$ \\
\hline LASr-c & 0.999 & $0.997-1.000$ & $<0.001$ & 0.998 & $0.995-0.999$ & $<0.001$ \\
\hline LAScd-c & 0.982 & $0.947-0.994$ & $<0.001$ & 0.982 & $0.945-0.994$ & $<0.001$ \\
\hline LASct-c & 0.996 & 0.989-0.999 & $<0.001$ & 0.997 & 0.992-0.999 & $<0.001$ \\
\hline
\end{tabular}

ICC, intraocular correlation coefficient; Cl, Confidence interval; LA VpreA, volume at onset of left atrial contraction; LA EF, left atrial ejection fraction; LASr, left atrial reservoir strain; LAScd, left atrial longitudinal strain during the conduit phase; LASct, left atrial contraction strain; LASr-c, left atrial reservoir circumferential strain; LAScd-c, left atrial conduit circumferential strain; LASct-c, left atrial contraction circumferential strain.

LASr-c, LAScd-c, and LASct-c. However, there was no significant difference in LA volume between the 2 groups in our study. LASct-c $>-4.5 \%$ could differentiate between high-risk and low-risk patients, with an AUC of 0.95 $(\mathrm{P}<0.0001)$. Furthermore, LASct-c exhibited high efficacy in the diagnosis of stroke, with an AUC of $0.957(\mathrm{P}<0.001)$. Therefore, strain parameters are more sensitive than volume parameters in distinguishing between thromboembolism risk in patients with NVAF. Moreover, we found LASct-c to be an independent factor for the identification of patients with NVAF who were at high risk of thromboembolism, and it was also found to be a marker for predicting the occurrence of stroke.

There are 3 phases of normal LA activity: the reservoir, conduit, and contraction phases (17). The specific temporal activity of the LA and the corresponding strain curve are as follows: After ventricular ED, the LA fills and stretches, and its strain curve increases, reaching the peak at the ventricular end-systolic (ES) phase (i.e., before the MV opening); this phase is the reservoir period of the LA. After $\mathrm{ES}$, the mitral valve opens, and the LA empties quickly until its pressure is equal to that of the left ventricle; this phase is the conduit period of the LA. Then, the strain decreases and the LA contracts, further expelling blood into the left ventricle, and the strain further decreases (18); this phase is the contraction period of the LA (Figure 6).

The 4D Auto LAQ tool was specially designed for the study of the LA. It can analyze longitudinal as well as circumferential LAS, and evaluate changes in strain parameters of LA activity in different phases (Figure 6). Due to the lack of a dedicated software for LAS measured by STE, software for LV analysis has also been applied in the study of LA pattern strains. The accuracy and repeatability of LAS measurements are affected by factors such as the thinwalled structure of the LA, irregular arrangement of LA myocytes (13), oval foramen on the atrial septum, and the 4 pulmonary vein openings on the LA wall among others.

The presence of AF leads to LA remodeling, which is characterized by LA enlargement/dysfunction caused by atrial interstitial fibrosis (19). This remodeling can weaken the LA reservoir, conduit, systolic function, and even result in the loss of systolic function (20). Saha et al. reported that the global LAS, as measured by STE, decreased proportionally to the CHADS 2 risk score (21). This observation implies that the larger the number of risk factors in patients with NVAF, the more severe the impairment of LA functions. Our findings were similar for thromboembolism risk in patients with NVAF based on the $\mathrm{CHA}_{2} \mathrm{DS}_{2}$-VASC score. Compared to the low-risk group, the thromboembolism high-risk group had significantly impaired LASr, LASct, LASr-c, LAScd-c, and LASct-c. We established that LASct-c is an independent factor for identifying patients with NVAF who are at high risk of thromboembolism. It also has a certain significance for guiding the use of anticoagulation therapy in patients with NVAF. We also found that LASct-c is highly effective in the diagnosis of stroke. This may be because LASCT-c is an indicator of LA systolic function; therefore, it has higher 


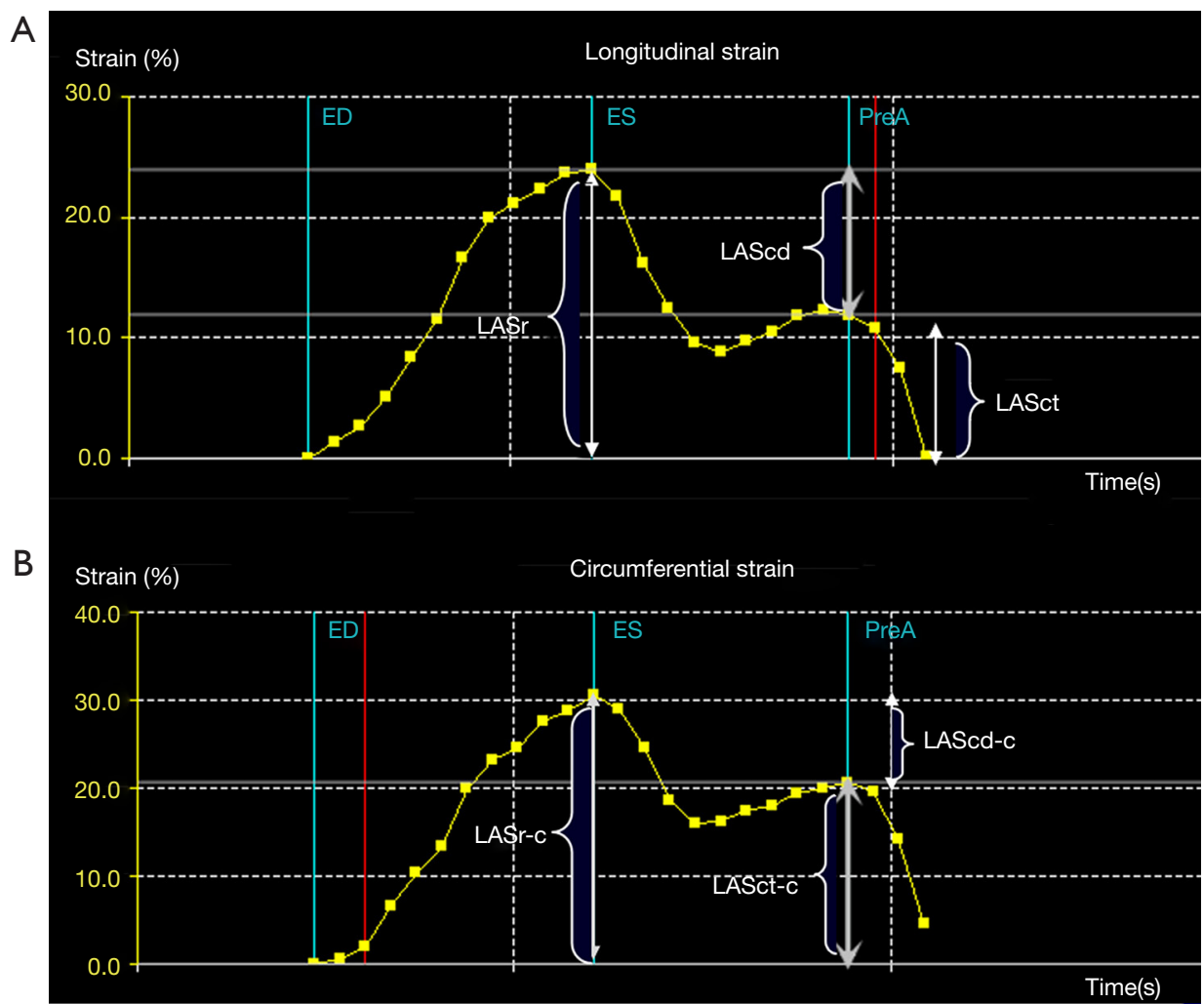

Figure 6 Strain relationship between 3 phases of left atrial activity. (A) shows the relationships among longitudinal strain parameters of the left atrium, and (B) shows the relationships among circumferential strain parameters of the left atrium.

sensitivity than the other strain parameters.

The most serious effect of AF on LA function occurs in the LA systolic phase (22), in which LA contractility is decreased due to atrial fibrosis and loss of electrical activity $(13,23,24)$. Our previous study revealed that LA reservoir strain in the stroke group was lower than that in the nonstroke group (25), with impaired LAS being independently correlated with stroke (26). Obokata et al. reported that LAS, as measured by STE, provides an incremental value to the $\mathrm{CHA}_{2} \mathrm{DS}_{2}$-VASc score in the prediction of stroke in patients with NVAF (27). Furthermore, compared to the LA volume, measurement of LAS can be used to detect LA functions. Kuppahally et al. found that LAS and the strain rate, as measured by STE, were significantly correlated with atrial fibrosis (28). Similarly, they did not report any significant differences in LA volume parameters between Paroxysmal AF group and Persistent AF group. These studies have shown that LAS is a promising index; however, its measurement is made via STE, which has various limitations and cannot be used to obtain the circumferential strain value. However, when LAS is measured by 4D Auto
LAQ, these limitations can be overcome. Further evaluation is needed to determine the value of LAS measured by 4D Auto LAQ in predicting stroke, and whether LASct-c is a better predictor of stroke than $\mathrm{CHA}_{2} \mathrm{DS}_{2}$-VASc.

The measurement of LAS by 4D Auto LAQ is extremely promising. LASct-c $=-4.5$ is the cut-off value for high risk and low risk of thromboembolism in patients with NVAF, and LASct-c shows high diagnostic efficacy for stroke. In conclusion, 4D Auto LAQ is a promising novel method for studying LA structure and functions.

\section{Study limitations}

This was a single-center, retrospective study. Since our sample size involved only 85 participants, some of our results may have been attributable to chance, and therefore need to be confirmed in a large-sample prospective study.

The LASct-c was found to be closely associated with stroke, hypertension, diabetes, and age $\geq 65$ years old in the $\mathrm{CHA}_{2} \mathrm{DS}_{2}$-VASc score; therefore, it was concluded that LASct-c $>-4.5 \%$ is completely equivalent to 
thromboembolism high risk, which cannot be completely determined.

\section{Conclusions}

LAS measured by 4D Auto LAQ is a useful index for the dynamic evaluation of LA function in patients with NVAF, and has higher sensitivity and accuracy than LA volume. LASct-c is independently associated with a high risk of thromboembolism and an LASct-c $>-4.5 \%$ may be of guiding value for the use of anticoagulant therapy in patients with NVAF.

\section{Acknowledgments}

We thank Dr. Dan Liu and Dr. Junpei Li for their professional advice in the preparation of this article.

Funding: This study was funded by the Science and Technology Research Project of Jiangxi Provincial Department of Education (grant no. GJJ190002), and the research topic of Teaching Reform Research Subject of Jiangxi Province (grant no. JXJG-19-1-40).

\section{Footnote}

Conflicts of Interest: All authors have completed the ICMJE uniform disclosure form (available at http://dx.doi. org/10.21037/qims-20-1381). The authors have no conflicts of interest to declare.

Ethical Statement: The authors are accountable for all aspects of the work in ensuring that questions related to the accuracy or integrity of any part of the work are appropriately investigated and resolved. The procedures used conformed to the standards of the World Medical Association Declaration of Helsinki (as revised in 2013). This study was approved by the Ethics Committee of the second Affiliated Hospital of Nanchang University, China. All participants provided verbal informed consents.

Open Access Statement: This is an Open Access article distributed in accordance with the Creative Commons Attribution-NonCommercial-NoDerivs 4.0 International License (CC BY-NC-ND 4.0), which permits the noncommercial replication and distribution of the article with the strict proviso that no changes or edits are made and the original work is properly cited (including links to both the formal publication through the relevant DOI and the license). See: https://creativecommons.org/licenses/by-nc-nd/4.0/.

\section{References}

1. Haeusler KG, Tütüncü S, Schnabel RB. Detection of Atrial Fibrillation in Cryptogenic Stroke. Curr Neurol Neurosci Rep 2018;18:66.

2. Zimetbaum P. Atrial Fibrillation. Ann Intern Med 2017;166:ITC33-48. Erratum in: Ann Intern Med 2017;166:920.

3. Sulzgruber P, Wassmann S, Semb AG, Doehner W, Widimsky P, Gremmel T, Kaski JC, Savarese G, Rosano GMC, Borghi C, Kjeldsen K, Torp-Pedersen C, Schmidt TA, Lewis BS, Drexel H, Tamargo J, Atar D, Agewall S, Niessner A. Oral anticoagulation in patients with nonvalvular atrial fibrillation and a CHA2DS2-VASc score of 1: a current opinion of the European Society of Cardiology Working Group on Cardiovascular Pharmacotherapy and European Society of Cardiology Council on Stroke. Eur Heart J Cardiovasc Pharmacother 2019;5:171-80.

4. January CT, Wann LS, Calkins H, Chen LY, Cigarroa JE, Cleveland JC Jr, Ellinor PT, Ezekowitz MD, Field ME, Furie KL, Heidenreich PA, Murray KT, Shea JB, Tracy CM, Yancy CW. 2019 AHA/ACC/HRS Focused Update of the 2014 AHA/ACC/HRS Guideline for the Management of Patients With Atrial Fibrillation: A Report of the American College of Cardiology/ American Heart Association Task Force on Clinical Practice Guidelines and the Heart Rhythm Society in Collaboration With the Society of Thoracic Surgeons. Circulation 2019;140:e125-51.

5. Walters TE, Kalman JM, Patel SK, Mearns M, Velkoska E, Burrell LM. Angiotensin converting enzyme 2 activity and human atrial fibrillation: increased plasma angiotensin converting enzyme 2 activity is associated with atrial fibrillation and more advanced left atrial structural remodelling. Europace 2017;19:1280-7.

6. Pinho-Gomes AC, Reilly S, Brandes RP, Casadei B. Targeting inflammation and oxidative stress in atrial fibrillation: role of 3-hydroxy-3-methylglutaryl-coenzyme a reductase inhibition with statins. Antioxid Redox Signal 2014;20:1268-85.

7. Yu HT, Lee JS, Kim TH, Uhm JS, Joung B, Hong GR, Lee MH, Shim CY, Pak HN. Advanced Left Atrial Remodeling and Appendage Contractile Dysfunction in Women Than in Men Among the Patients With Atrial Fibrillation: Potential Mechanism for Stroke. J Am Heart 
Assoc 2016;5:e003361.

8. Habibi M, Zareian M, Ambale Venkatesh B, Samiei S, Imai M, Wu C, Launer LJ, Shea S, Gottesman RF, Heckbert SR, Bluemke DA, Lima JAC. Left Atrial Mechanical Function and Incident Ischemic Cerebrovascular Events Independent of AF: Insights From the MESA Study. JACC Cardiovasc Imaging 2019;12:2417-27.

9. Collier P, Phelan D, Klein A. A Test in Context: Myocardial Strain Measured by Speckle-Tracking Echocardiography. J Am Coll Cardiol 2017;69:1043-56.

10. Chu M, Qian L, Zhu M, Yao J, Xu D, Chen M. Circumferential strain rate to detect lipopolysaccharideinduced cardiac dysfunction: a speckle tracking echocardiography study. Quant Imaging Med Surg 2019;9:151-9.

11. Voigt JU, Pedrizzetti G, Lysyansky P, Marwick TH, Houle H, Baumann R, Pedri S, Ito Y, Abe Y, Metz S, Song JH, Hamilton J, Sengupta PP, Kolias TJ, d'Hooge J, Aurigemma GP, Thomas JD, Badano LP. Definitions for a common standard for 2D speckle tracking echocardiography: consensus document of the EACVI/ ASE/Industry Task Force to standardize deformation imaging. J Am Soc Echocardiogr 2015;28:183-93.

12. Sasaki S, Watanabe T, Tamura H, Nishiyama S, Wanezaki M, Sato C, Yamaura G, Ishino M, Arimoto T, Takahashi H, Shishido T, Miyamoto T, Kubota I. Left atrial strain as evaluated by two-dimensional speckle tracking predicts left atrial appendage dysfunction in patients with acute ischemic stroke. BBA Clin 2014;2:40-7.

13. Donal E, Lip GY, Galderisi M, Goette A, Shah D, Marwan M, et al. EACVI/EHRA Expert Consensus Document on the role of multi-modality imaging for the evaluation of patients with atrial fibrillation. Eur Heart J Cardiovasc Imaging 2016;17:355-83.

14. Sugimoto T, Robinet S, Dulgheru R, Bernard A, Ilardi F, Contu L, et al. Echocardiographic reference ranges for normal left atrial function parameters: results from the EACVI NORRE study. Eur Heart J Cardiovasc Imaging 2018;19:630-8.

15. Zoghbi WA, Adams D, Bonow RO, Enriquez-Sarano M, Foster E, Grayburn PA, Hahn RT, Han Y, Hung J, Lang RM, Little SH, Shah DJ, Shernan S, Thavendiranathan P, Thomas JD, Weissman NJ. Recommendations for Noninvasive Evaluation of Native Valvular Regurgitation: A Report from the American Society of Echocardiography Developed in Collaboration with the Society for Cardiovascular Magnetic Resonance. J Am Soc Echocardiogr 2017;30:303-71.
16. Nielsen PB, Skjøth F, Overvad TF, Larsen TB, Lip GYH. Female Sex Is a Risk Modifier Rather Than a Risk Factor for Stroke in Atrial Fibrillation: Should We Use a CHA(2) DS(2)-VA Score Rather Than CHA(2)DS(2)-VASc? Circulation 2018;137:832-40.

17. Hoit BD. Left atrial size and function: role in prognosis. J Am Coll Cardiol 2014;63:493-505.

18. Cameli M, Mandoli GE, Loiacono F, Sparla S, Iardino E, Mondillo S. Left atrial strain: A useful index in atrial fibrillation. Int J Cardiol 2016;220:208-13.

19. Kallergis EM, Manios EG, Kanoupakis EM, Mavrakis $\mathrm{HE}$, Arfanakis DA, Maliaraki NE, Lathourakis CE, Chlouverakis GI, Vardas PE. Extracellular matrix alterations in patients with paroxysmal and persistent atrial fibrillation: biochemical assessment of collagen type-I turnover. J Am Coll Cardiol 2008;52:211-5.

20. Providência R, Trigo J, Paiva L, Barra S. The role of echocardiography in thromboembolic risk assessment of patients with nonvalvular atrial fibrillation. J Am Soc Echocardiogr 2013;26:801-12.

21. Saha SK, Anderson PL, Caracciolo G, Kiotsekoglou A, Wilansky S, Govind S, Mori N, Sengupta PP. Global left atrial strain correlates with CHADS2 risk score in patients with atrial fibrillation. J Am Soc Echocardiogr 2011;24:506-12.

22. Manning WJ, Silverman DI, Katz SE, Douglas PS. Atrial ejection force: a noninvasive assessment of atrial systolic function. J Am Coll Cardiol 1993;22:221-5.

23. Schotten U, Verheule S, Kirchhof P, Goette A. Pathophysiological mechanisms of atrial fibrillation: a translational appraisal. Physiological reviews 2011;91:265-325.

24. Cui Q, Wang H, Zhang W, Wang H, Sun X, Zhang Y, Yang H. Enhanced left atrial reservoir, increased conduit, and weakened booster pump function in hypertensive patients with paroxysmal atrial fibrillation. Hypertens Res 2008;31:395-400.

25. Leung M, van Rosendael PJ, Abou R, Ajmone Marsan N, Leung DY, Delgado V, Bax JJ. Left atrial function to identify patients with atrial fibrillation at high risk of stroke: new insights from a large registry. Eur Heart J 2018;39:1416-25.

26. Shih JY, Tsai WC, Huang YY, Liu YW, Lin CC, Huang YS, Tsai LM, Lin LJ. Association of decreased left atrial strain and strain rate with stroke in chronic atrial fibrillation. J Am Soc Echocardiogr 2011;24:513-9.

27. Obokata M, Negishi K, Kurosawa K, Tateno R, Tange S, Arai M, Amano M, Kurabayashi M. Left atrial strain 
provides incremental value for embolism risk stratification over CHA2DS2-VASc score and indicates prognostic impact in patients with atrial fibrillation. J Am Soc Echocardiogr 2014;27:709-16.e4.

28. Kuppahally SS, Akoum N, Burgon NS, Badger TJ, Kholmovski EG, Vijayakumar S, Rao SN, Blauer J,

Cite this article as: Chen L, Zhang C, Wang J, Guo L, Wang X, Liu F, Li X, Zhao Y. Left atrial strain measured by 4D Auto LAQ echocardiography is significantly correlated with high risk of thromboembolism in patients with non-valvular atrial fibrillation. Quant Imaging Med Surg 2021;11(9):39203931. doi: 10.21037/qims-20-1381
Fish EN, Dibella EV, Macleod RS, McGann C, Litwin SE, Marrouche NF. Left atrial strain and strain rate in patients with paroxysmal and persistent atrial fibrillation: relationship to left atrial structural remodeling detected by delayed-enhancement MRI. Circ Cardiovasc Imaging 2010;3:231-9. 